a small amount of dichloro-acetic acid, separated and identified as its aniline salt, showing that the course of the reaction is probably similar to that when ammonia is not present, but it is evident that the reaction is more complicated, probably by reason of secondary reactions in which ammonia takes part.

\title{
Summary
}

'The reactions of trichloro-acetic acid and of ethyl trichloro-acetate with copper have been studied. Diethyl tetrachloro-succinate has been prepared by the action of copper with ethyl trichloro-acetate. It boils at $156^{\circ}$ and $13 \mathrm{~mm}$. pressure. Tetrachloro-succinic acid is formed in small amount by the action of copper with trichloro-acetic acid alone or in ether solution. The acid was not obtained in pure condition. Aniline tetrachloro-succinate was obtained, m. p. 149-150 ${ }^{\circ}$.

Dichloro-acetic acid is formed in satisfactory quantity when trichloroacetic acid reacts with copper powder in benzene or aqueous solution, and this reaction is recommended as a means of preparation of dichloroacetic acid in any desired quantity.

Evidence is presented from which it seems probable that these reactions involve the formation of an intermediate relatively unstable compound in which copper is joined directly to carbon, and that the course of the reaction is determined by the manner in which the copper is eliminated from this intermediate compound. The suggestion is made that the action of copper as a condensing agent, as used by Ullmann, Sandmeyer and Gattermann, may involve the formation of similar intermediate compounds.

AMHERST, MASSACHUSETTS

[Contribution from the Laboratory of Pharmace, OF WASHINGTON]

\section{THE ACTION OF NITROSYL CHLORIDE ON NORMAL HEPTANE}

\author{
By E. V. LyNN AND O. HIITON
}

Received December 15, 1921

It has recently been found by one of us $^{1}$ that nitrosyl chloride will readily react with normal heptane in the sunlight, a reaction which is apparently general for the paraffin hydrocarbons. From a preliminary study of the products it was assumed that dipropyl ketone is the chief one, but no satisfactory proof was given. In the present work, we have repeated the former investigation and demonstrated the correctness of the assumptions made then as to the course of the reaction.

Since hydrogen chloride is developed in quantity, the assumption was made that the other product is a nitroso compound. All representatives of this class are known to possess a deep blue color; hence the formation I.Lynn, This Journal, 41, 368 (1919). 
of this color in the present reaction seems evidence enough, considering the raw materials used. The disappearance of this color and the precipitation of a yellow oil pointed to a change of the nitroso compound to an oxime, since it is well known that in this class of substances, unless the nitroso group is connected to a tertiary carbon atom, there is an unvarying tendency to rearrangement. A further substantiation of this is the ready hydrolysis of the product to form hydroxylamine and a volatile substance, a characteristic behavior of the oximes. We may, therefore, cxpress the changes as

$$
\begin{aligned}
& \mathrm{C}_{7} \mathrm{H}_{16}+\mathrm{NOCl} \longrightarrow \mathrm{C}_{7} \mathrm{H}_{15} \mathrm{NO}+\mathrm{HCl} \\
& \mathrm{C}_{7} \mathrm{H}_{15} \mathrm{NO} \longrightarrow \mathrm{C}_{7} \mathrm{H}_{14} \mathrm{NOH} \\
& \mathrm{C}_{7} \mathrm{H}_{14} \mathrm{NOH}+\mathrm{H}_{2} \mathrm{O} \longrightarrow \mathrm{C}_{7} \mathrm{H}_{14} \mathrm{O}+\mathrm{NH}_{2} \mathrm{OH}
\end{aligned}
$$

Ammonium chloride and hydroxylamine were isolated at various times as products of the reaction, the former undoubtedly being a decomposition product of the latter under the conditions of the experiment.

The volatile product was isolated in larger quantity and identified as chiefly di-n-propyl ketone, the other components being probably the remaining ketones from normal heptane. Furthermore, the oxime was purified and examined, its physical constants being those of butyronoxime.

\section{Experimental}

The normal heptane ${ }^{2}$ had been distilled from the oleoresin of Pinus Jeffreyi and was further purified by shaking it repeatedly with sulfuric acid and a subsequent distillation over sodium hydroxide. Pure nitrosyl chloride was easily prepared by passing the gases from the reaction of sodium nitrite and hydrochloric acid into sulfuric acid and gently heating the product with dry sodium chloride. Crude nitrosyl chloride was made by heating a mixture of 4 volumes of hydrochloric acid and 1 volume of nitric acid, the product as obtained consisting principally of nitrosyl chloride and chlorine. The crude reagent gave the same reactions with heptane as did the pure, so that it was of no advantage to use the first method, and the crude mixture with chlorine was employed throughout.

This mixture of nitrosyl chloride and chlorine was led into heptane until the latter was thoroughly saturated, as indicated by a very dark red-brown color. The solution was then placed directly in the sunlight, the result being an immediate evolution of large quantites of hydrogen chloride which continued for about one-half hour. Sometimes samples appeared to be boiling vigorously. Accompanying the evolution of hydrogen chloride was a decided color change from orange or reddishbrown to green or blue, the shade depending upon the degree of saturation; the color usually obtained with highly saturated solutions was a verdant or emerald green. Also, at this stage minute globules of an oil, usually light brown but sometimes dark, made their appearance.

After the reaction had proceeded thus far, hydrogen chloride ceased

${ }^{2}$ Secured through the courtesy of the Wisconsin Pharmaceutical Experiment Station. 
to be given off so vigorously, the color gradually faded to either lighter shades of green or Copenhagen blue, the quantity of precipitated oil increased, and finally the color disappeared entirely, an evident sign of complete reaction. The heptane was then saturated, as previously described.

The resultant oil, light colored at first, rapidly became dark reddishbrown. It remained acid in reaction, slowly evolving hydrogen chloride and, when warm or at ordinary temperature, precipitated small quantities of feathery crystals of ammonium chloride, but when cooler a mixture of anmonium chloride and hydroxylamine hydrochloride was formed.

When $214 \mathrm{~g}$. of oil had been collected, a portion $(81 \mathrm{~g}$.) was neutralized with barium carbonate to free it from hydrogen chloride, the oil was extracted with chloroform, and the chloroform evaporated. The dark brown oily residue was subjected to fractional distillation, the portions boiling at $80^{\circ}$ to $140^{\circ}, 140^{\circ}$ to $180^{\circ}$, and $180^{\circ}$ to $197^{\circ}$ being collected. During the last part of the process because of the presence of a small amount of free hydrogen chloride, there was considerable decomposition, accompanied by deposition of ammonium chloride and much foaming. In spite of this, the third fraction was comparatively pure butyronoxime, as shown by the physical constants.

\begin{tabular}{lccl} 
& $\begin{array}{c}\text { Boiling point } \\
\text { c.. }\end{array}$ & Specific gravity & \multicolumn{1}{c}{ Index of refraction } \\
Butyronoxime $^{3} \ldots \ldots$ & 193 & $0.8924\left(20^{\circ} / 4\right)$ & $1.4475\left(20^{\circ}\right)$ \\
Third Fraction. . . . & $180-197$ & 0.9257 & $1.444\left(14.5^{\circ}\right)$
\end{tabular}

The remaining portion of the oil $(133 \mathrm{~g}$.) was distilled with steam, a process which completely hydrolyzed the oxime. The ketone product thus obtained was a colorless or slightly yellow liquid with an aromatic odor, very volatile, comparatively soluble in water, and with a burning acrid taste. It was dried over anhydrous calcium chloride ${ }^{4}$ and fractionated twice, giving the following fractions: up to $130^{\circ}, 12 \mathrm{cc}$; $130-150^{\circ}, 35 \mathrm{cc}$.; $150-160^{\circ}, 5 \mathrm{cc}$; and a residue of $5 \mathrm{cc}$. Practically all of the second fraction came over between $140^{\circ}$ and $150^{\circ}$. The physical constants of this fraction compared with those of dipropyl ketone are as follows.

\begin{tabular}{lccc} 
& \multicolumn{1}{c}{$\begin{array}{c}\text { Boiling point } \\
\text { C. }\end{array}$} & \multicolumn{1}{c}{ Specific gravity } & \multicolumn{1}{c}{ Index of refraction } \\
Di- $n$-propyl ketone ${ }^{5}$. & 143.52 & $0.8210\left(14.8^{\circ} / 14.8\right)$ & $1.40732\left(22^{\circ}\right)$ \\
Fraction 2 $\ldots \ldots \ldots$ & $140-150$ & $0.83826\left(15.6^{\circ} / 15.6\right)$ & $1.4125\left(14.5^{\circ}\right)$
\end{tabular}

This shows fairly conclusively that the main portion consists of di- $n$ propyl ketone with the probable admixture of a small amount of its isomers.

This was further identified by conversion into the semicarbazone.

3 Trapnensojanz, Ber., 26, 1433 (1893).

- Curiously, calcium chloride is soluble in cold dipropyl ketone, but very slightly in the hot substance.

${ }^{5}$ Louginine, Ann. chim., [7] 13, 331 (1898); Perkin, J. Chem. Soc., 69, 1236 (1896); Brubl, J. prakt. Chem., [2] 50, 141 (1894). 
After recrystallization from high-boiling gasoline, this had a melting point of $117^{\circ}$, compared with $123^{\circ}$ for the semicarbazone from synthetic dipropyl ketone. A mixture of the two gave approximately the same melting point.

The ketone was also oxidized with potassium dichromate to give butyric and propionic acids. The oxidation mixture was distilled with steam fractionally and the fractions analyzed by means of the silver salts in the usual way. The results show the presence of butyric acid in the first and of propionic acid in the later fractions:

Silver butyrate: $\mathrm{Ag}, 55.4$; silver propionate: $\mathrm{Ag}, 59.7$; oxidation fractions; $\mathrm{Ag}$. $54.34,54.80,57.82,58.80$.

Since the results were obtained but slowly at best, an effort was made to substitute ultra-violet rays for sunlight and, also, to increase the speed of the reaction by an increase in temperature. Repeated trial has shown that the formation of nitroso compound (blue color) takes place under the influence of sunlight, at temperatures up to $80^{\circ}$, or with ultra-violet light. For some reason, however, the less refrangible rays of the spectrum are necessary to effect final conversion of the nitroso compound into the oxime.

\section{Summary}

Nitrosyl chloride reacts with normal heptane in the sunlight to give di-n-propyl nitroso-methane, which rearranges to form dipropyl ketoxime.

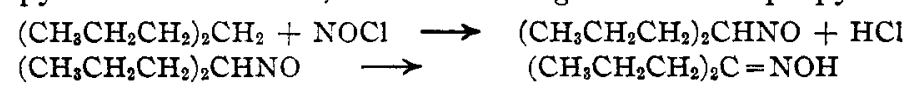

Upon distillation with steam the oxime is hydrolyzed to form di-n-propyl ketone and hydroxylamine.

$\left(\mathrm{CH}_{3} \mathrm{CH}_{2} \mathrm{CH}_{2}\right)_{2} \mathrm{C}=\mathrm{NOH}+\mathrm{H}_{2} \mathrm{O} \longrightarrow\left(\mathrm{CH}_{3} \mathrm{CH}_{2} \mathrm{CH}_{2}\right)_{2} \mathrm{C}=\mathrm{O}+\mathrm{NH}_{2} \mathrm{OH}$

There is no record in the literature of any process in which nitrosyl chloride acts upon the paraffin hydrocarbons; hence this reaction furnishes an entirely new line of investigation. Since petroleum ether and gasoline behave similarly it is reasonable to suppose that the reaction is general for this series of hydrocarbons. Furthermore, there is every reason to expect that nitrosyl chloride will react in the same way with other compounds containing an alkyl residue. We are at present engaged in investigating the action on toluene, cymene, etc., and will report the results shortly.

SEATTLE. WASHINGTON 